\title{
Applications of Artificial Intelligence and ML in Business
}

\author{
N. Thirupathi Rao ${ }^{1}$ and Debnath Bhattacharyya ${ }^{2}$ \\ Department of Computer Science and Engineering, Vignan's Institute of Information \\ Technology, Visakhapatnam-530049, AP, India \\ Inakkathiru@gmail.com,2debnathb@gmail.com
}

\begin{abstract}
The utilization of artificial intelligence and its related techniques is going in rapid level of growth. As the technologies are upgrading from time to time, the technologies are also increasing the utilization of these techniques such that to provide the more sophisticated facilities to the users. The utilization of various applications of artificial intelligence includes face recognition, palm recognition and other applications etc. As the technology trends going, the utilization is also increasing and in the current paper, thee applications are given with a brief details. Various sectors where the current AI techniques are utilizing in a very high growth to be noted and presented in detail in the current paper. This growth has to be observed in various fields and all those areas were discussed in detail.
\end{abstract}

Keywords: Artificial intelligence, Machine learning, Face recognition, Smart technologies, Business applications, Marketing applications, E-commerce applications

\section{Introduction}

Artificial intelligence is the recently developing technologies and it is helping the other technologies in the market and society to achieve good levels of the technology. The technology is growing day by day with the usage of these techniques [1]. This utility has changed the entire worlds prospective towards the artificial intelligence and its related areas of working and support. With this utility, the business of the various big companies in the world had entered into the he level of very few millions of dollars to the billions of dollars for their businesses. The utility of these techniques and its other related areas will give the best outputs when compared to the other sets of implementations. The techniques that can be used for the research experiments and other issues, the results will be very impressive and will not be expected by the users or the scientists. The results astonish the scientists who are working on these areas.

The utilization of these applications started form the initiation of using rule based mechanisms. In rule based mechanisms, the data with which we had decided to work with the machine will be given in the format of rules. The machine will work on the basis of these rules and will take the decisions appropriately. At first, the data that was available for the currently considered problem will be collected from various sources. All this data will be converted in to the form of rules with various answers for the same rules. All these rules will be stored in the database of the intelligent machine or the artificial intelligent machine where the data will be used by the machine for taking the decisions on the currently raised problems. The place where these setoff rules will be stored can be taken as the knowledge base [2].

Article history:

Received (March 30, 2019), Review Result (May 15, 2019), Accepted (July 9, 2019) 
This database can be treated as the knowledge base due to the reason that these machines will use this database such that to take decisions based on the rules available in the database or the knowledge base. Now, the task is to identify the appropriate rule that was similar to that of the currently considered problem was the big task or the big challenge. Several algorithms and techniques are being using today such that to increase the accuracy of finding the similar set of rules in the database and try to identify the appropriate solution for the current problem that was raised in the current scenario. The utility of these rule based systems are growing day by day [3]Now a days, these rule based systems are also to be taken as the expert systems. These expert systems can be used in various fields such that to find the best outputs from the existing scenarios.

The various areas are like the medical applications, agricultural areas and other related areas etc. The farmers or the poor people cannot afford the lab tests or the increasing level of the laboratory tests. In such cases, the usage of these artificial intelligence based systems or the expert systems will be very much helpful for identifying the diseases and giving the best suggestions or the cure suggestions from time to time. The poor people and the farmers who cannot afford for suggestions from various experts and other scientists, these expert systems which will help them easily and freely by using the online and internet facilities. Now a day, the growth of the internet and smartphones, the utility of these technologies had increased a lot and benefitted the customers and the people from the society in a huge manner [4].

By using these advanced applications and other related areas, the performance of the machines and applications had increased a lot. The algorithms utilization had increased a lot and the performance will be at high speed rates and the performance at the time of speed and accuracy will be more and higher at ground level applications. The people from the society are getting more and more benefits and other applications in a better way for their better life. The applications of these techniques and area will be discussed in detail in the following sections.

\section{Some of the applications of artificial intelligence and its related areas}

In the current section, we had discussed the various set of applications where the artificial intelligence and its related techniques were sued in the current developing technologies and trends in the current society [6][7]. Some of these applications are,

Business applications

Chatbots

E-commerce

Healthcare

Cyber Security

Banking

Commercial Applications

\subsection{Business applications}

Now a day, artificial intelligence was not theory, it is practical and it is being implemented and tested for various applications in businesses and the results are getting amazing. Many practical applications are using these technologies very highly in these days. The companies started using the AI globally from last five years and it is growing in number by day to day. This number is increasing in such a way those we cannot he predict the future utility of this aspect. As a result, the business people are getting more and more profits and more security to the employees and the expensive costs can be reduced to a good level. 


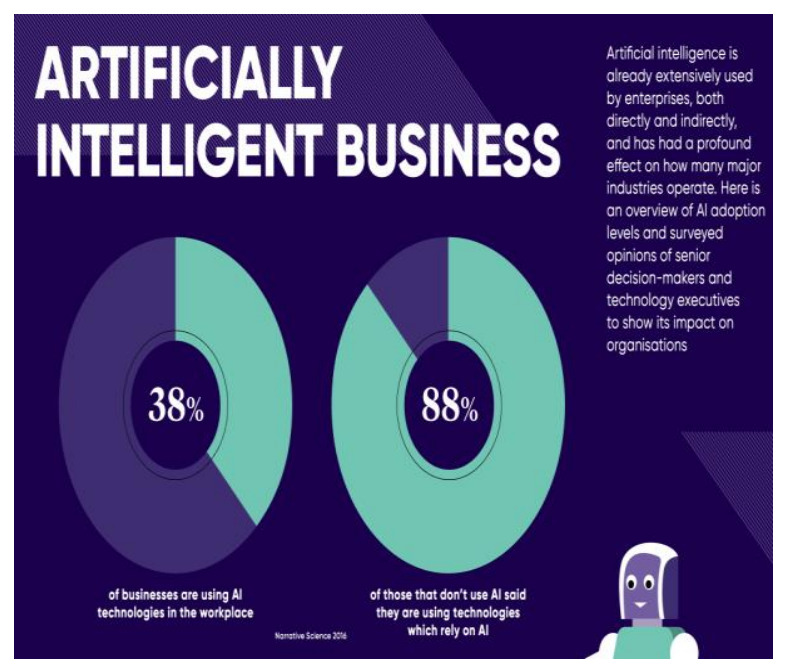

Figure 1. An example of business applications with AI [2]

Now a days, all the companies globally are planning to implement the AI technologies and techniques in their companies such that to reduce their expenditures and good productivity and further cutting costs. The security to the employees and other benefits to the employees which are being spent for the human beings can be replaced by these machines in a good countable manner. As a result of that, the companies are going for the trend of utilizing these techniques and trying to get more benefits and trying to reduce their expenditure costs[5] .

\subsection{Chatbots}

The usage of current technology applications and other algorithms was increasing and it is used in almost all the smart phones releasing now a days. The usage had increased a lot for various applications in the smartphones too. It is also started using in other areas like healthcare, gaming etc. The chatting applications and other people discussing applications with the help of smartphones with internet facility had increased a lot. The processing of such applications and other related issues can be resolved by using some good algorithms such that o store such data and processing of such data was required. The processing includes storing of data, retrieving of data and finding the similar type of words which were typed by the users previously in the previous chats.

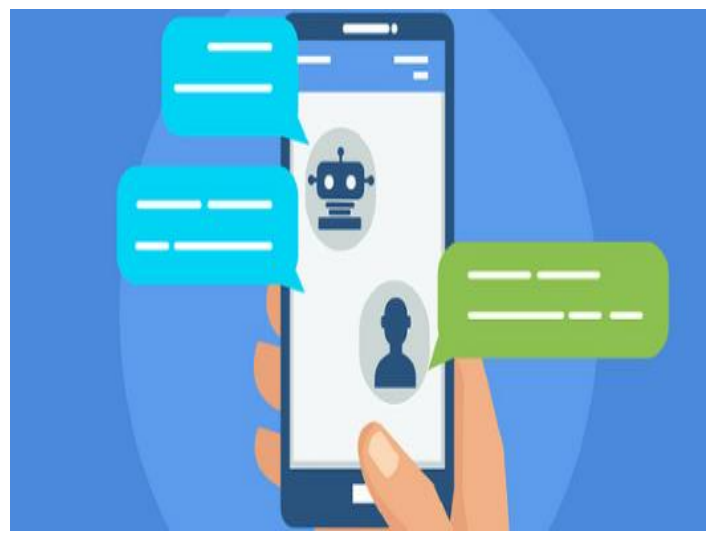

Figure 2. Chatbots example of AI applications [1] 
The utility of these applications had included the voice based chatting and voice based commands also designed such that to process the applications and tasks to be performed by the users words or the users voice. It is going to be further more and further to next levels by observing the user's behaviour or the users face expressions.

\subsection{Artificial intelligence in ecommerce}

The implementation and utility of AI and its related techniques and other algorithms had increased a lot for getting better outputs and better businesses for the ecommerce companies and websites. Now days, the ecommerce applications and business websites had increased a lot such that the big companies around the worked are trying to enter in to this business and investing more and more. With this technology, the customers are getting the facilities and sources in such a way to their door to door delivery and choosing the things from the home. These companies and websites are providing the customers with good offers and discounts such that to attract the more number of customers from time to time.

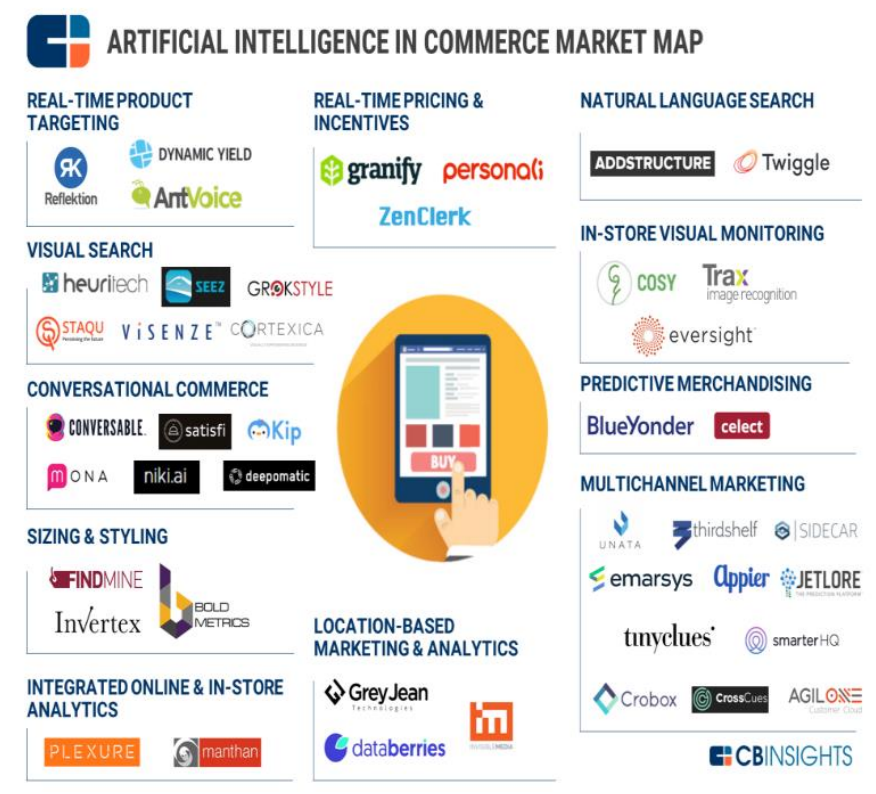

Figure 3. Ecommerce applications using AI [1]

The utilization of AI techniques in the ecommerce or the business applications and their websites will gives the data to the customers such that to find their size, colour or the shape of the products can be identified. Without any hassles, the customers can find the similar set of objects which they had already observed and shopped earlier to this. Whenever, there is a change in the price of those particular objects, the users will get the updates of such data immediately to the customers with the updated data. The visual related trends and borders of these AI techniques are being increasing from time to time.

\subsection{AI to improve workplace communication}

In the current section, the application of $\mathrm{AI}$ and its related techniques and methods were discussed in detail and the performance of these techniques in the work places was discussed 
in detail in the current sections. In the present days, the business applications and their communication is full of applying the content with news applications, channels for both news and entertainment, tools for online software's and other online applications for financial and other applications, and also to observe the working nature of the employees in a industry. The utilization of $\mathrm{AI}$ and its technique sin the business applications such that the business will be increased in a good manner. Allowing the people in the business will get good amount of profits and that will lead to the employees for getting good hikes and other benefits too. As a result, the companies and industries can concentrate on their performance in terms of productivity and focus on the productivity such that either to enhance or to decrease the productivity of the organization.

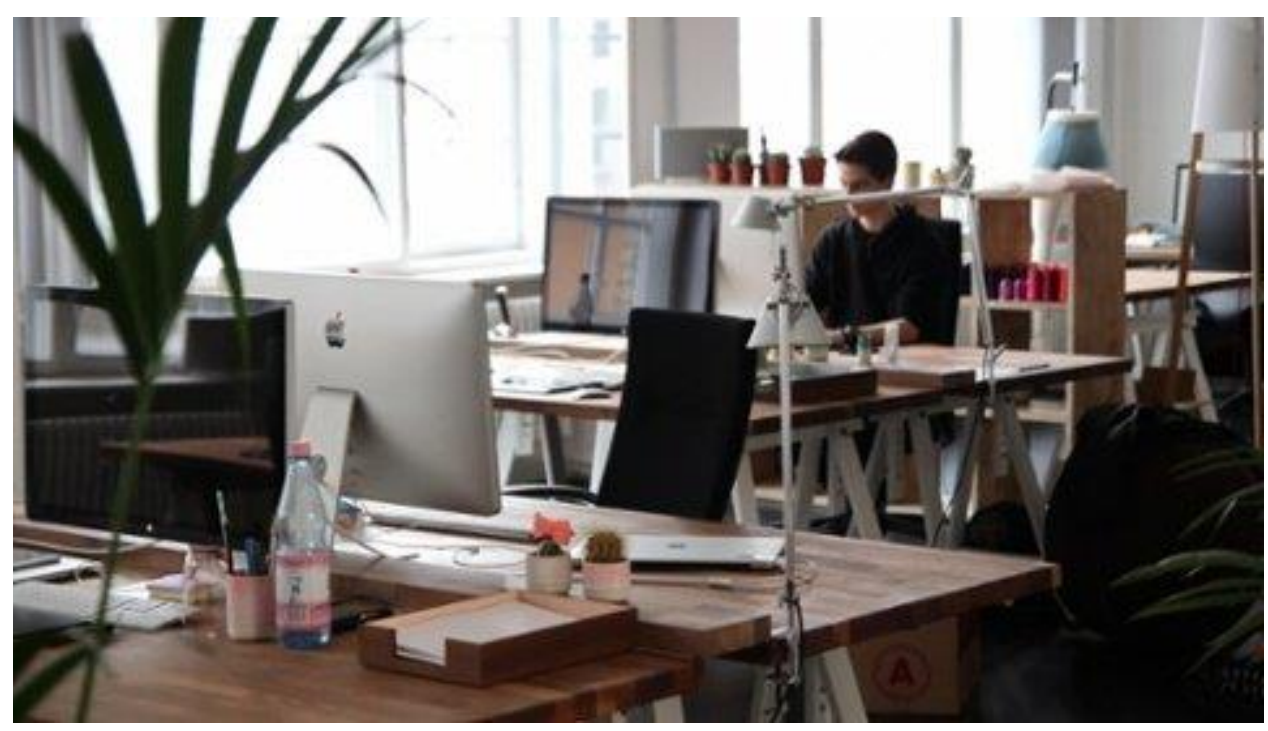

Figure 4. An example of workplace implementation of AI techniques [1][3]

With the utilization of AI techniques in the work place, the users and the employees can understand the actual works which were completed already and the works which takes some more time to implement. The major goal in any industry was to identify the working hours and the working time where the organizations will get the maximum time to perform the tasks and can save more profits and benefits. The utilization of time effectively and efficiently is more important task and consideration to be taken and it should be keen for implementation and success of the current trends in the markets. In the same way, the identification and utilization of the resources for the current application trends is also important to be considered.

\subsection{AI in healthcare}

The utilization of artificial intelligence and its related techniques in the field of medical and health care, drugs testing was increasing day by day. The identification of diseases in the human beings was becoming easy day to day due to the utilization of AI and its related techniques. The diagnosis of diseases is becoming easy due to the utilization of these techniques with additional techniques of algorithms. With the utilization of these techniques, the severe diseases like the cancer, diarrhoea, heart problems, diabetes and other severe diseases. Several tests will be conducted in the laboratories for identifying the diseases in the initial phase or the medium level or in the severe level of the diseases. The tests will be made more easy and accurate due 
to the utilization of AI techniques. The machinery will use the previous tests data of previous patients such that to identify the diseases more accurately. The precision in finding the diseases with the help of the previous data in the database will helpful such that to identify the diseases more accurately and more precisely.

Now a days, almost all the hospitals and the medical laboratories are maintaining the full database of the patients who were coming for the hospitals for the treatment and other related issues. This stored data will be useful to the patients in the future purposes for their visits and also $t$ identify the development in the health of the patient with the previous visit of the patient to the hospital to the current visit of the patient to the same hospital. At the same time, the doctors also will have the clear picture of the patient's condition with the previous visit with the current visit. Also the doctors can have the clear picture of the problems to be faced in the coming future with the current state of the problems with the patient.

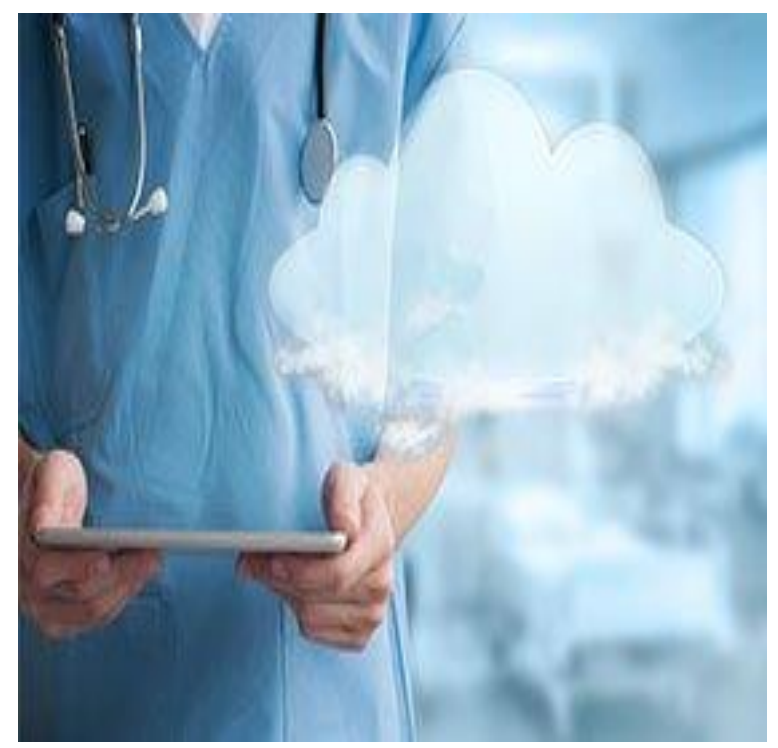

Figure 5. Healthcare application example of AI [1]

The utilization of these AI techniques will help us in getting the patients disease data in accurate manner and the predicted data or the result can be trusted for almost it is true as it will consider the data for the previous patient's data as much as possible. The data that was available in the hospital is always made softcopy from time to time and it will be stored for further processing or for further detailed analysis such that to identify the actual disease details in correct manner. The identification of these genetics or the disease and the utility of the drugs for various diseases were also important. The testing of the drugs and their quality is also important and also identifying the level of the working of the drug was also important to identify the patient's dosage levels. The detailed dosage levels should be identified in the standard process level such that to identify the patients situations and the disease stage of the patient. If the correct dosage of the drug was not given, it may leads to the death of the patients in some cases. Hence, it is always a good practice to identify the correct dosage of drugs to the patients for their appropriate diseases. 


\subsection{Banking}

Now days, all the banks in the world wide are trying to adapt the Artificial Intelligence and its related techniques and other algorithms. All the banks are trying to adapt these techniques due to the facilities and the provisions these algorithms and other techniques are providing for the development of banks. These methods had created a great impact on the banking and other related sectors for further increasing of the banks and their businesses. The banks are looking for the automation of the banking processes and other banking related all types of transactions. By the implementation of these transactions, the maintenance costs of the banks and other costs were becoming reduced day to day and the banks are increasing their profits day by day. Hence, all the banks are trying to reduce the capital amount on the investment on various sectors or various applications and tasks in the nationalized and international banks.

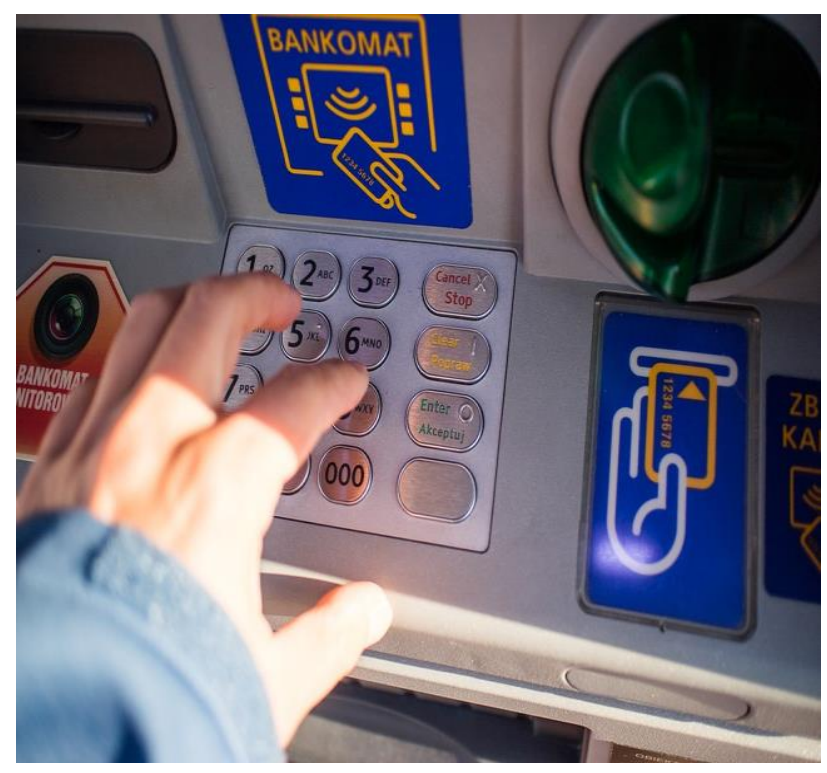

Figure 6. Banking applications example using AI [3]

The implementation of various applications and other technologies to be incorporated in the various processes and other applications that were being utilized in the current trends of the societies and other banks. The implementation of these techniques and other algorithms in the banking applications was incorporated in the payments and transactions that were taking place in the current markets today. By making the transactions in this way, the banks are also trying to reduce their operating costs and trying to improve their profits. The banks are trying to implement the latest trends and latest technologies in the market for the customers to provide various applications and other facilities with more security for their banking applications and other transactions. As the banks are charging much charges for providing such facilities, the business applications and other money for the banks ion these transactions is increasing day to day.

\subsection{Commercial applications}

The other important and considerable area where the AI applications are using highly was the ecommerce. The business applications and their related areas was the ecommerce where almost all the big companies and investors around the world are investing huge amounts. The 
big companies are investing huge amounts for getting good set of profits. Now a day, this ecommerce business is increasing in such a way, that the growth was very high and good. Some of the good applications or the companies where this ecommerce business was growing very fast was the Amazon, flipkart etc. This two ecommerce companies are occupying the most of the business through online in recent days. The data was collected from the users by registering their data, their hobbies, their birthdays and other days and it will be stored in their databases. The likes of the customers and the items which they had purchased earlier and list $f$ items which they previously verified or visited will be given the high priority and it will be stored Whenever, there is a slight change or slight changes in the prices of such articles, the immediate message will be generate dot the customers for their information. In the customer is interested, he can go through the website and can book or order the item.

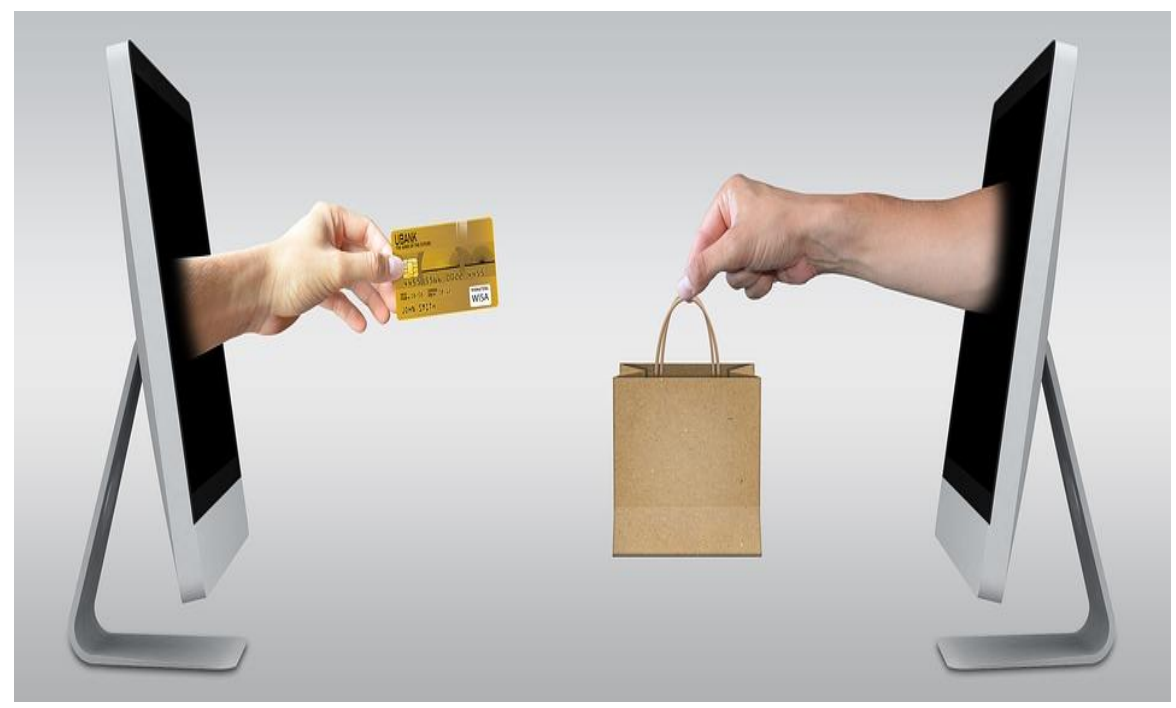

Figure 7. Ecommerce application example using AI Techniques [3]

The other set of advantages and other facilities that the users can achieve from these websites will be the application of websites for these items can reduce the operating costs of such items. If the same thing will be placed in a showroom, the maintenance costs will be reduced. If it will be in the showroom, the power costs, employee's costs and other maintenance costs will be more. If they maintain the websites, the same thing will be reduced in some aspects and the other costs will be reduced. The owner people can maintain only the websites data and the financial transactions also will be completed early and other type of benefits will be available to the customers. The people are moving towards such businesses due to the sophisticated facilities like the easy to choose the items without any hassle, more amount time for taking the decisions, checking the availability of items and other benefits.

\section{Conclusions}

In the present paper, the applications of artificial intelligence and other techniques to be implemented in the various aspects was discussed in detail. The utilization of AI techniques and algorithms had increased a lot and other sets of applications, where these techniques and other methods were being implementing successfully and that leading to the better services to the society. Various sectors in the society are getting good benefits with the utilization of these techniques in the current market and the current trends in the society. 


\section{References}

[1] Newgenapps, "Top 11 uses \& applications of artificial intelligence \& Ml in business," published on January, https://www.newgenapps.com/blog/ai-uses-applications-of-artificial-intelligence-ml-business, (2018)

[2] CodeWave technologies, "3 Practical applications of AI for businesses, that can be implemented right away," (2018)

[3] Learnitude Technologies, "Applications of artificial intelligence in top 10 areas “, April, (2018)

[4] Barton D., Woetzel J., Seong J. and Tian Q., “Artificial intelligence: implications for China,” McKinsey Global Institute, Discussion Paper presented at 2017 China Development Forum, pp.1-14, (2017)

[5] Brynjolfsson E. and Mcafee A., "The business of artificial intelligence," Harvard Business Review published on December, https://hbr.org/cover-story/2017/07/the-business-of-artificial-intelligence, (2017)

[6] Jia K. and Kenney M., "Mobile internet business models in china: vertical hierarchies, horizontal conglomerates, or business groups," BRIE Working Paper 2016-6, pp.1-8, (2016)

[7] Kenney M. and Zysman J., "The rise of the platform economy," Issues in Science and Technology, vol.32, no.3, pp.61-63, (2016) 
Applications of Artificial Intelligence and ML in Business

This page is empty by intention. 